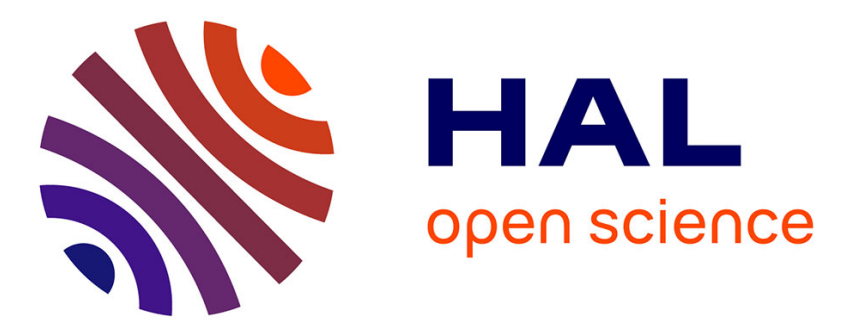

\title{
Elliptic Curves Associated with Simplest Quartic Fields Sylvain Duquesne
}

\section{To cite this version:}

Sylvain Duquesne. Elliptic Curves Associated with Simplest Quartic Fields. Journal de Théorie des Nombres de Bordeaux, 2007, 19 (1), pp.81-100. 10.5802/jtnb.575 . lirmm-00145846

\section{HAL Id: lirmm-00145846 https://hal-lirmm.ccsd.cnrs.fr/lirmm-00145846}

Submitted on 11 May 2007

HAL is a multi-disciplinary open access archive for the deposit and dissemination of scientific research documents, whether they are published or not. The documents may come from teaching and research institutions in France or abroad, or from public or private research centers.
L'archive ouverte pluridisciplinaire HAL, est destinée au dépôt et à la diffusion de documents scientifiques de niveau recherche, publiés ou non, émanant des établissements d'enseignement et de recherche français ou étrangers, des laboratoires publics ou privés. 


\title{
Elliptic curves associated with simplest quartic fields
}

\author{
par SYLVAIN DUQUESNE
}

\begin{abstract}
RÉSumÉ. Nous étudions la famille infinie des courbes elliptiques associées aux "simplest quartic fields". Si le rang de telles courbes vaut 1 , nous déterminons la structure complète du groupe de Mordell-Weil et nous trouvons tous les points entiers sur le modèle original de la courbe. Notons toutefois que nous ne sommes pas capables de les trouver sur le modèle de Weierstrass quand le paramètre est pair. Nous obtenons également des résultats similaires pour une sous-famille infinie de courbes de rang 2. A notre connaissance, c'est la première fois que l'on a autant d'information sur la structure du groupe de Mordell-Weil et sur les points entiers pour une famille infinie de courbes de rang 2. Le principal outils que nous avons utilisé pour cette étude est la hauteur canonique.

Abstract. We are studying the infinite family of elliptic curves associated with simplest cubic fields. If the rank of such curves is 1 , we determine the whole structure of the Mordell-Weil group and find all integral points on the original model of the curve. Note however, that we are not able to find them on the Weierstrass model if the parameter is even. We have also obtained similar results for an infinite subfamily of curves of rank 2 . To our knowledge, this is the first time that so much information has been obtained both on the structure of the Mordell-Weil group and on integral points for an infinite family of curves of rank 2 . The canonical height is the main tool we used for that study.
\end{abstract}

\section{Introduction}

In [4], we studied elliptic curves associated with simplest cubic fields. In the case of curves of rank 1, we determined both the structure of the Mordell-Weil group and all integral points. Several questions remained unanswered at the end of this study. Is it possible to do the same work with other families of rank 1 curves? Is it possible to generalize to families of curves of higher ranks? Xavier Roblot and Franck Leprevost suggested that I should work on elliptic curves associated with simplest quartic fields. This family has several interesting properties. 
- There is an explicit point on every curve of the family, which is a necessary condition for the kind of study we are interested in.

- Contrary to simplest cubic fields, the curves are not torsion-free. Hence we can check if the method used in [4] is also valid when there are torsion points.

- It is possible to extract a subfamily of curves of rank at least 2 with two explicit points.

In this paper, we will first see that the method used for simplest cubic fields to determine the structure of the Mordell-Weil group can also be used for simplest quartic fields. It can also be generalized to higher ranks and probably to other families. However, we will see that this is not the case for integral points, even though a technical trick enabled us to conclude in our case.

Finally recent papers $([2],[3])$, not known when this paper was written, would be helpful in simplifying some of the calculations. They provide better bounds than those used in this paper and then will probably eliminate some cases which are done by hand in the following.

\section{Simplest quartic fields}

The term "simplest" has been used to describe certain number fields defined by a one-parameter family of polynomials. The regulator of these simplest fields is small in an asymptotic sense, so their class number tends to be large. This is why they have generated so much interest. In degree 4 , simplest quartic fields are defined by adjoining to $\mathbb{Q}$ a root of the polynomials

$$
X^{4}-t X^{3}-6 X^{2}+t X+1
$$

where $16+t^{2}$ is not divisible by an odd square (which ensures the irreducibility of the polynomial). These fields were studied, among other things, by Gras, who proved that this family is infinite [5]. Later, Lazarus studied their class number $[8,9]$. More recently, they were studied by Louboutin [10], Kim [7] and Olajos [11].

\section{Elliptic curves associated with simplest quartic fields}

In the following, we are interested in the infinite family of elliptic curves $Q_{t}$ given by the equation

$$
Y^{2}=X^{4}-t X^{3}-6 X^{2}+t X+1,
$$

where $16+t^{2}$ is not divisible by an odd square. The discriminant is $\Delta_{t}=2^{6}\left(16+t^{2}\right)^{3}$.

Let us first put the curve into the Weierstrass form

$$
C_{t}: y^{2}=x^{3}-\left(16+t^{2}\right) x
$$


by sending the point $[0,1]$ to infinity using the transformation $\varphi$

$$
\begin{aligned}
& x=\frac{2 Y-2 X^{2}+t X+2}{X^{2}}, \\
& y=\frac{\left(Y+X^{2}+1\right)\left(2 Y-2 X^{2}+t X+2\right)}{X^{3}} .
\end{aligned}
$$

Such curves are special cases of curves defined by equations of the form $y^{2}=x^{3}+D x$ which often appear in the literature. For instance they are studied in the book of Silverman [14] where several general results are proved, one of which is given below

Proposition 3.1. Let $D$ be a fourth-power-free integer. Let $E_{D}$ be the elliptic curve defined over $\mathbb{Q}$ by the equation

$$
y^{2}=x^{3}+D x .
$$

If $D \neq 4$ and $-D$ is not a perfect square, then

$$
E_{D}(\mathbb{Q})_{\text {tors }} \simeq \mathbb{Z} / 2 \mathbb{Z}
$$

This result can be applied to our family.

Corollary 3.2. Let $t$ be an integer defining a simplest quartic field. The only torsion points on $C_{t}(\mathbb{Q})$ are the point at infinity and the 2-torsion point $[0,0]$. The torsion points on $Q_{t}(\mathbb{Q})$ can be obtained using the inverse map of $\varphi$.

As usual with elliptic curves, we are interested in the following two Diophantine problems.

(1) Determination of the structure of the Mordell-Weil group $Q_{t}(\mathbb{Q})$ (or equivalently $C_{t}(\mathbb{Q})$ ). This means that we want to compute the torsion subgroup (already done thanks to Proposition 3.1), the rank and a set of generators for the free part.

(2) Determination of all integral points on both $Q_{t}$ and $C_{t}$, since a famous theorem of Siegel states that there are only finitely many such points.

Concerning the second problem, it is important to note that the integral points are dependent on the model. In the case of elliptic curves associated with simplest quartic fields, both models $\left(Q_{t}\right.$ and $\left.C_{t}\right)$ given above are interesting. Nevertheless they are linked thanks to the following property.

Proposition 3.3. Let $t$ be an integer defining a simplest quartic field and $[X, Y]$ be an integral point on the quartic model. Then $\varphi([X, Y])+[0,0]$ is an integral point on the cubic model.

Proof. It is easy to formally compute $\varphi([X, Y])+[0,0]$ using the group law on $C_{t}(\mathbb{Q})$.

$\varphi([X, Y])+[0,0]=\left[2 Y+2 X^{2}-t X-2,-\left(Y+X^{2}+1\right)\left(2 Y+2 X^{2}-t X-2\right)\right]$

which proves the proposition. 
This means that it is sufficient to find all integral points on $C_{t}$ in order to find those of $Q_{t}$. On the other hand, the structure of the Mordell-Weil group does not depend on the model, so we will work on $C_{t}$ in the following.

\section{Experimental approach}

Using the magma algebra system, we performed a large number of computations both of the structure of the Mordell-Weil group and of the integral points. Here we do not present the results we obtained, but we give the most important observations we deduced from these computations.

(1) The rank is never 0.

(2) The rank parity only depends on the congruence class of $t$ modulo 16.

(3) The point $[-4,2 t]$ can always be in a system of generators of $C_{t}(\mathbb{Q})$.

(4) In the case of rank 1 , the only integral points on $C_{t}$ are $[0,0],[-4, \pm 2 t]$ and $\left[\frac{t^{2}}{4}+4, \pm\left(\frac{t^{3}}{8}+2 t\right)\right]$ if $t$ is even.

(5) In the case of rank $1,[0, \pm 1]$ are the only integral points on $Q_{t}$.

(6) In higher ranks, there are very few integral points on $Q_{t}$ apart from a point with a $x$-coordinate equal to -3 .

The first observation is trivial to prove. Indeed, $[-4,2 t]$ is always a point on $C_{t}(\mathbb{Q})$. Moreover, we already proved that $[0,0]$ and the point at infinity are the only torsion points. So $[-4,2 t]$ has an infinite order and $C_{t}(\mathbb{Q})$ has a rank of at least one.

\section{The sign of the functional equation}

We will now prove the second observation assuming the conjecture of Birch and Swinnerton-Dyer.

Theorem 5.1. Let $t$ be an integer defining a simplest quartic field. Assuming the Birch and Swinnerton-Dyer conjecture, the Mordell-Weil rank of $C_{t}(\mathbb{Q})$ is even if and only if

$$
t \equiv 0, \pm 1, \pm 7 \bmod 16 \text {. }
$$

Proof. We use the sign of the functional equation which is 1 if and only if the rank is even assuming the conjecture of Birch and Swinnerton-Dyer. This sign can be computed as a product of local signs.

$$
\varepsilon=\varepsilon_{\infty} \prod_{p \text { prime }} \varepsilon_{p}
$$

The value of the sign at the Archimedean place is always $\varepsilon_{\infty}=-1$. Concerning finite places, the local sign depends on the type of curve reduction. It can be computed using the tables given by Rizzo in [12]. The places 2 and 3 must be treated separately. The first remark is that $16+t^{2}$ is never 
divisible by 3 , so 3 is always a prime of good reduction and $\varepsilon_{3}=1$.

Now let $p$ be a prime number greater than or equal to 5 . Hereafter in this paper, $v_{p}(x)$ will denote the $p$-adic valuation of $x$.

If $p \nmid \Delta_{t}$, then $\varepsilon_{p}=1$.

If $p \mid \Delta_{t}$, we have that $v_{p}\left(\Delta_{t}\right)=3$ since $16+t^{2}$ is not divisible by an odd square. In this case, Rizzo's tables give $\varepsilon_{p}=\left(\frac{-2}{p}\right)$, so

$$
\varepsilon_{p}=\left\{\begin{array}{rll}
(-1)^{\frac{p-1}{4}} & \text { if } & p \equiv 1 \bmod 4 \\
-(-1)^{\frac{p+1}{4}} & \text { if } & p \equiv-1 \bmod 4
\end{array}\right.
$$

We want now to compute the product of all these local signs.

Let $\delta_{t}=16+t^{2}$ and $\delta_{t}^{\prime}=\frac{\delta_{t}}{2^{v_{2}\left(\delta_{t}\right)}}$. Since $t$ defines a simplest quartic field, there are $k$ different prime numbers $p_{1}, \ldots, p_{k}$ which are congruent to 1 modulo 4 and $r$ different prime numbers $p_{k+1}, \ldots, p_{k+r}$ which are congruent to -1 modulo 4 , such that

$$
\delta_{t}^{\prime}=p_{1} \ldots p_{k} p_{k+1} \ldots p_{k+r} .
$$

Moreover, it is easy to prove that $\delta_{t}^{\prime}$ equals 1 modulo 4 , so $r$ must be even. Let $q_{i}=\frac{p_{i}-1}{4}$ if $i \leq k$ and $q_{i}=\frac{p_{i}+1}{4}$ if $i \geq k+1$. We have

$$
\begin{aligned}
\delta_{t}^{\prime} & =\left(1+4 q_{1}\right) \ldots\left(1+4 q_{k}\right)\left(-1+4 q_{k+1}\right) \ldots\left(-1+4 q_{k+r}\right) \\
& \equiv 1+4 q_{1}+\cdots+4 q_{k+r} \bmod 8 .
\end{aligned}
$$

On the other hand,

$$
\begin{aligned}
\prod_{p \neq 2} \varepsilon_{p} & =(-1)^{q_{1}} \ldots(-1)^{q_{k}}(-1)^{r}(-1)^{q_{k+1}} \ldots(-1)^{q_{k+r}} \\
& =(-1)^{q_{1}+\cdots+q_{k+r}}
\end{aligned}
$$

So

It is easy to deduce that

$$
\prod_{p \neq 2} \varepsilon_{p}=(-1)^{\frac{\delta_{t}^{\prime}-1}{4}}
$$

$$
\prod_{p \neq 2} \varepsilon_{p}=1 \Longleftrightarrow t \text { odd or } t \equiv 0 \bmod 16 \text { or } t \equiv \pm 4 \bmod 32 .
$$

We will now compute the local sign $\varepsilon_{2}$. For this, we again use the tables of Rizzo. For each value of $t$ modulo 32, the 2-adic valuations of both $\Delta_{t}$ and the usual invariant $c_{4}=3.2^{4}\left(16+t^{2}\right)$ give the value of $\varepsilon_{2}$. We have

$$
\varepsilon_{2}=1 \Longleftrightarrow t \equiv \pm 3 \bmod 8 \text { or } t \equiv \pm 4 \bmod 32 \text {. }
$$

We just have to multiply $\varepsilon_{\infty}, \prod_{p \neq 2} \varepsilon_{p}$ and $\varepsilon_{2}$ to achieve the proof of the theorem. 
Remark. We chose to use the tables of Rizzo instead of those of Halberstadt [6] because the minimality of the model is not required. In fact, the model is minimal if $t$ is not divisible by 4 . When $t$ is divisible by 4 , the minimal model is $y^{2}=x^{3}-\left(1+t^{2}\right) x$.

We now want to prove the observations 3,4 and 5. For this, we use a method similar to that we used for elliptic curves associated with simplest cubic fields in [4]. The central part of this method is a good estimate of the canonical height. Let us briefly review this canonical height.

\section{Canonical height on elliptic curves}

Even though it is possible to work on number fields, we will restrict our study to $\mathbb{Q}$ since this is the case we are interested in. Let $E$ be an elliptic curve defined over $\mathbb{Q}$ and $P=[x, y]$ be a point on $E(\mathbb{Q})$. If $x=n / d$ with $\operatorname{gcd}(n, d)=1$ the naïve height of point $P$ is defined as

$$
h(P)=\max (\log |n|, \log |d|) .
$$

This height function is the main tool for the proof of the Mordell-Weil theorem which states that $E(\mathbb{Q})$ is finitely generated. The naïve height has some nice properties but we need a more regular function. This function is the canonical height and is defined as follows

$$
\hat{h}(P)=\lim _{k \rightarrow \infty} \frac{h(k P)}{k^{2}}=\lim _{n \rightarrow \infty} \frac{h\left(2^{n} P\right)}{4^{n}} .
$$

Remark. The canonical height is sometimes defined as half of this value, so one must be very careful which definition is used for results from different origins.

The canonical height has a lot of interesting properties. We will just mention here those that we will use later in this work.

(1) We have

$$
\hat{h}(P)=0 \Longleftrightarrow P \in E(\mathbb{Q})_{\text {tors }} .
$$

(2) Function $\hat{h}$ is a quadratic form on $E(\mathbb{Q})$.

(3) Let

$$
\langle P, Q\rangle=\frac{\hat{h}(P+Q)-\hat{h}(P)-\hat{h}(Q)}{2},
$$

denote the scalar product associated with $\hat{h}$. If $P_{1}, \ldots, P_{n}$ are $n$ points in the free part of $E(\mathbb{Q})$, let us define the elliptic regulator of points $P_{i}$ by

$$
R\left(P_{1}, \ldots, P_{n}\right)=\operatorname{det}\left(\left\langle P_{i}, P_{j}\right\rangle\right)_{1 \leq i, j \leq n} .
$$

Then, points $P_{1}, \ldots, P_{n}$ are linearly independent if and only if their elliptic regulator is not equal to zero. 
The naïve height is much easier to compute than the canonical height, so it is interesting to have explicit bounds for the difference between both of them. Such bounds are given by Silverman in [16].

Theorem 6.1 (Silverman). Let $E$ be an elliptic curve defined over $\mathbb{Q}$. Let $\Delta$ be the discriminant of $E$ and $j$ its $j$-invariant. Then for any $P$ in $E(\mathbb{Q})$ we have

$$
-\frac{h(j)}{4}-\frac{h(\Delta)}{6}-1.946 \leq \hat{h}(P)-h(P) \leq \frac{h(j)}{6}+\frac{h(\Delta)}{6}+2.14
$$

However, better bounds on the canonical heights are required for our purpose. For instance, if the naïve height of $P$ is small, the lower bound given by Silverman does not give any information since $\hat{h}(P)$ is always nonnegative. We will now briefly recall two ways to compute the canonical height. Both will be used hereafter in this work to improve Silverman's bounds for the curves we are interested in.

\section{Computation of the canonical height}

The two ways of computation we will present here consist of expressing the canonical height as a sum of local functions. The finite part of the height equals 0 for all primes of good reduction. For primes of bad reduction, it can be computed using a technical but simple algorithm given in [1].

The main part of the computation is focused on the Archimedean contribution which we will denote $\hat{h}_{\infty}$. This can be done by two ways. The first one uses $q$-expansions and consists of evaluation of the following formula

$$
\hat{h}_{\infty}(P)=\frac{1}{16} \log \left|\frac{\Delta}{q}\right|+\frac{1}{4} \log \left(\frac{y(P)^{2}}{\lambda}\right)-\frac{1}{2} \log \theta,
$$

where, if $\omega_{1}$ and $\omega_{2}$ denote the periods of the curve, and $z(P)$ is the elliptic logarithm of the point $P$

$$
\begin{aligned}
\lambda & =\frac{2 \pi}{\omega_{1}}, \\
q & =e^{2 \imath \pi \frac{\omega_{1}}{\omega_{2}}} \\
\theta & =\sum_{n=0}^{\infty}(-1)^{n} q^{\frac{n(n+1)}{2}} \sin ((2 n+1) \lambda \Re e(z(P)))
\end{aligned}
$$

If the curve is explicitly given, this method is very efficient since the series $\theta$ converges rapidly. However, we are dealing with a family of elliptic curves and, in this context, computation of the terms of the series $\theta$ seems difficult. We can still give an upper bound for this series. It is indeed trivial that

$$
|\theta| \leq \frac{1}{1-|q|} .
$$


This will provide a lower bound for the canonical height which is more useful than that given by Theorem 6.1. Such a lower bound combined with Silverman's upper bound was successfully used for simplest cubic fields in [4]. Concerning simplest quartic fields, these bounds can also be used when the rank of $C_{t}(\mathbb{Q})$ is 1 . However, they are not sharp enough when the rank is 2 . The second way of computing the Archimedean contribution will provide these better bounds.

This other way is slower but more appropriate for specific cases we are studying. It was developed by Tate and was improved by Silverman in [15]. It consists of computing the simple series

$$
\hat{h}_{\infty}(P)=\log |x(P)|+\frac{1}{4} \sum_{n=0}^{\infty} \frac{c_{n}}{4^{n}}
$$

where $c_{i}$ are easily computable and bounded. The main advantage is that the computation of the $c_{i}$ of a specific point can be done even for our family whereas computation of the terms of the series $\theta$ seems difficult for a family. Moreover, Silverman gives bounds for the error term if only $N$ terms are used in the series. Let $H=\max \left(4,2|a|, 4|b|, a^{2}\right)$, then

$$
\hat{h}_{\infty}(P)=\log |x(P)|+\frac{1}{4} \sum_{n=0}^{N-1} \frac{c_{n}}{4^{n}}+R(N),
$$

with

$$
\frac{1}{3.4^{N}} \log \left(\frac{\Delta^{2}}{2^{60} H^{8}}\right) \leq R(N) \leq \frac{1}{3.4^{N}} \log \left(2^{11} H\right) .
$$

Thus, Tate's method will provide better bounds for the canonical height of specific points, such as $[-4,2 t]$. However, we first need bounds which are valid for any point in $C_{t}(\mathbb{Q})$, so we use Silverman's theorem and $q$ expansions.

\section{Approximation of the canonical height of any point on $C_{t}(\mathbb{Q})$}

As explained above, an upper bound of the canonical height is given by Silverman's theorem:

$$
\hat{h}(P)-h(P) \leq \frac{h(j)}{6}+\frac{h(\Delta)}{6}+2.14 .
$$

Applying this bound to our family gives the following proposition.

Proposition 8.1. Let $t$ be an integer defining a simplest quartic field. Let $P$ be a point in $C_{t}(\mathbb{Q})$, then

$$
\hat{h}(P) \leq h(P)+\frac{1}{2} \log \left(16+t^{2}\right)+4.08
$$


We will now use the decomposition of the canonical height as a sum of local functions to obtain a lower bound.

The first step involves the computation of the finite part of the canonical height of a point $P=\left[\frac{a}{d^{2}}, \frac{b}{d^{3}}\right]$. For this, we follow the algorithm given in [1]. If $p$ is an odd prime number, it is easy to prove that the local contribution at $p$ is $2 \log \left(p^{v_{p}(d)}\right)-\frac{1}{2} \log (p)$ if $p$ divides $a, b$ and $16+t^{2}$ and 0 otherwise. The contribution at 2 is more difficult to find since there are several cases depending on the 2-adic valuation of $t$ and $a$. We summarize the result in the following table.

\begin{tabular}{|c|c|}
\hline condition & contribution at 2 \\
\hline$d$ even & $2 \log \left(2^{v_{2}(d)}\right)$ \\
$t$ odd and $a$ odd & $-\frac{1}{2} \log (2)$ \\
$t$ even and $a$ odd or $a$ even and $t$ odd & 0 \\
$v_{2}(a)=1$ and $v_{2}(t)=1$ & $-\frac{3}{2} \log (2)$ \\
$v_{2}(a)=1$ and $v_{2}(t) \geq 2$ or $v_{2}(t)=1$ and $v_{2}(a) \geq 2$ & $-\log (2)$ \\
$v_{2}(a)=2$ and $v_{2}(t)=2$ or $v_{2}(a) \geq 3$ and $v_{2}(t) \geq 3$ & $-2 \log (2)$ \\
$v_{2}(a)=2$ and $v_{2}(t) \geq 3$ or $v_{2}(t)=2$ and $v_{2}(a) \geq 3$ & $-\frac{5}{2} \log (2)$ \\
\hline
\end{tabular}

Finally, the local contribution at non-Archimedean places to the canonical height of any point $P$ is given by

$$
\hat{h}_{f}(P)=2 \log (d)-\frac{1}{2} \log \left(\begin{array}{c}
\prod p_{i} \\
p_{i} \neq 2, p_{i} \mid a, b, 16+t^{2}
\end{array}\right)+\hat{h}_{2}(P),
$$

where $\hat{h}_{2}(P)$ is equal to zero if $d$ is even and to the contribution at 2 , given in the previous table, if $d$ is odd.

The second step is the computation of the Archimedean contribution. As explained above, we will use $q$-expansions since we want a lower bound that is valid for any point on the curve. We first need approximations for the periods $\omega_{1}$ and $\omega_{2}$.

Lemma 8.2. Let $t$ be an integer defining a simplest quartic field and $C_{t}$ be the associated elliptic curve. Let $\omega_{1}$ and $\omega_{2}$ be the periods of $C_{t}$ such that $\omega_{1}$ and $\imath \omega_{2}$ are positive, then

$$
\omega_{1}=\imath \omega_{2} \text { and } \frac{\pi}{\sqrt{2}\left(16+t^{2}\right)^{\frac{1}{4}}} \leq \omega_{1} \leq \frac{\pi}{\left(16+t^{2}\right)^{\frac{1}{4}}}
$$

Proof. Let $\delta=\sqrt{16+t^{2}}$. The $C_{t}$ equation is

$$
y^{2}=x^{3}-\left(16+t^{2}\right) x=x(x-\delta)(x+\delta) .
$$


Thus, with the convention we chose for the periods, $\omega_{1}$ and $\omega_{2}$ are given by the integrals

$$
\begin{aligned}
& \omega_{1}=\int_{-\delta}^{0} \frac{1}{\sqrt{x(x-\delta)(x+\delta)}} \\
& \omega_{2}=\int_{0}^{\delta} \frac{1}{\sqrt{x(x-\delta)(x+\delta)}} .
\end{aligned}
$$

A trivial change of variable shows that $\omega_{1}=\imath \omega_{2}$.

Concerning $\omega_{1}$, within the integration range, we have $-2 \delta \leq x-\delta \leq-\delta$, so that

$$
\frac{1}{\sqrt{2}\left(16+t^{2}\right)^{\frac{1}{4}}} \int_{-\delta}^{0} \frac{1}{\sqrt{x(x+\delta)}} \leq \omega_{1} \leq \frac{1}{\left(16+t^{2}\right)^{\frac{1}{4}}} \int_{-\delta}^{0} \frac{1}{\sqrt{x(x+\delta)}} .
$$

The result follows thanks to an easy change of variables.

So, thanks to this lemma, we can give a lower bound for the Archimedean contribution to the canonical height of any point $P=\left[\frac{a}{d^{2}}, \frac{b}{d^{3}}\right]$ in the free part of $C_{t}(\mathbb{Q})$.

$$
\hat{h}_{\infty}([P]) \geq 0.38+\frac{1}{8} \log \left(16+t^{2}\right)+\frac{1}{2} \log \left(\frac{b}{d^{3}}\right) .
$$

Thus, combining this lower bound with the non-Archimedean contributions, we have

$\hat{h}(P) \geq 0.38-\frac{5}{2} \log (2)+\frac{1}{8} \log \left(16+t^{2}\right)+\frac{1}{2} \log (d)+\frac{1}{2} \log \left(\frac{b}{\prod_{p_{i} \neq 2, p_{i} \mid a, b, 16+t^{2}}}\right)$.

The last two terms are always positive, so this provides an explicit lower bound.

However, these terms can be used to reduce the constant $0.38-\frac{5}{2} \log (2)$. Let $g$ be the ged of $a, b$ and $16+t^{2}$ divided by its higher power of 2 . Let $A=\frac{a}{g}$ and $B=\frac{b}{g}$. With these notations, the sum of the last two terms of the lower bound equals $\frac{1}{2} \log (B d)$, so a lower bound for $B d$ will improve the lower bound for $\hat{h}(P)$.

Based on the fact that $\left[\frac{a}{d^{2}}, \frac{b}{d^{3}}\right]$ is a point on the curve, we prove that $g$ must satisfy the equation

$$
A^{3} g^{2}-B^{2} g-A\left(16+t^{2}\right) d^{4}=0 .
$$

Since $g$ is an integer, the discriminant of this degree 2 polynomial must be the square of an integer, say $C$, such that

$$
B^{4}+64 A^{4} d^{4}=\left(C-2 A^{2} t d^{2}\right)\left(C+2 A^{2} t d^{2}\right) .
$$


It is easy to deduce that, if such a $C$ exists, then

$$
t \leq \frac{B^{4}+64 A^{4} d^{4}-1}{4 A^{2} d^{2}}
$$

Let us assume that the local contribution at 2 is negative, i. e. $d$ is odd and $t$ and $a$ are together even or odd. In this case, we have $4 \mid B$ and $A \leq B^{2}$. If $B=4$ and $d=1$, the above condition becomes $t \leq 4160$. For all $t \leq 4160$ and $A \leq 16$, we can check if the discriminant of the degree 2 polynomial is a square. This never occurs if $t>256$. Thus, if $t>256$, it is not possible to have $B=4$ and $d=1$, so either $B \geq 4$ and $d \geq 3$ or $B \geq 8$ and $d=1$. In any case, $B d \geq 8$. We can now give a lower bound for the canonical height.

Proposition 8.3. Let $t$ be an integer greater than 256 defining a simplest quartic field. Let $P$ be any point in the free part of $C_{t}(\mathbb{Q})$. We have

$$
\begin{aligned}
& \hat{h}(P) \geq \quad 0.38+\frac{1}{8} \log \left(16+t^{2}\right) \quad \text { if } t \text { is odd, } \\
& \hat{h}(P) \geq 0.38+\frac{1}{8} \log \left(16+t^{2}\right)-\log (2) \quad \text { in any case. }
\end{aligned}
$$

Proof. If $t$ is odd and $\hat{h}_{2}(P)=0$ then $B d \geq 1$ is sufficient to give the required lower bound for $\hat{h}(P)$.

If $t$ is odd and $\hat{h}_{2}(P)<0$, this contribution is $-\frac{1}{2} \log (2)$ and we proved that $B d \geq 8$. This provides a better lower bound than required.

Finally, if $t$ is even and $\hat{h}_{2}(P)<0$, this contribution is greater than or equal to $-\frac{5}{2} \log (2)$ and we proved that $B d \geq 8$. Again, this is sufficient to conclude.

\section{Estimates of the canonical height of a specific point: $[-4,2 t]$}

The previous bounds are valid for any non-torsion point on $C_{t}(\mathbb{Q})$, so they provide bounds for the points $G_{1}=[-4,2 t]$. However, we need a more precise approximation for $\hat{h}\left(G_{1}\right)$. So we will use Tate's series to compute $\hat{h}\left(G_{1}\right)$ in terms of $t$. For our purpose, it is sufficient to compute the first four terms of the series, so

$$
\hat{h}_{\infty}\left(G_{1}\right)=\log (4)+\frac{1}{4}\left(c_{0}+\frac{c_{1}}{4}+\frac{c_{2}}{16}+\frac{c_{3}}{64}\right)+R(4) .
$$

We are using the algorithm given in [15] to formally compute $c_{0}, c_{1}, c_{2}$ and $c_{3}$. In fact, the only significant contribution comes from $c_{0}$. Thus we only give approximations for the others.

$$
c_{0}=2 \log \left(16+t^{2}\right)-8 \log (2) \text { and } 0 \leq c_{1}, c_{2}, c_{3} \leq \log (4) .
$$


Let us now estimate the error term $R(4)$. In the case of elliptic curves defined by simplest quartic fields, the constant $H$ involved in the approximation of the rest (1) equals $\left(16+t^{2}\right)^{2}$ so

$$
\begin{aligned}
& \frac{1}{3.4^{4}} \log \left(\frac{2^{12}\left(16+t^{2}\right)^{6}}{2^{60}\left(16+t^{2}\right)^{16}}\right) \leq R(4) \leq \frac{1}{3.4^{4}} \log \left(2^{11}\left(16+t^{2}\right)^{2}\right) \\
& -\frac{48 \log (2)+10 \log \left(16+t^{2}\right)}{768} \leq R(4) \leq \frac{11 \log (2)+2 \log \left(16+t^{2}\right)}{768} .
\end{aligned}
$$

Concerning non-Archimedean contributions, the only non-zero one comes from 2. The previous table can be used to estimate the contribution at 2

$$
\begin{cases}\hat{h}_{2}\left(G_{1}\right)=0 & \text { if } t \text { is odd } \\ -\frac{5}{2} \log (2) \leq \hat{h}_{2}\left(G_{1}\right) \leq-\log (2) & \text { otherwise. }\end{cases}
$$

Finally, combining these estimates we obtain an estimate for the canonical height of the point $[-4,2 t]$

$$
\begin{array}{ll}
\hat{h}([-4,2 t]) \geq \frac{187}{384} \log \left(16+t^{2}\right)-\frac{1}{16} \log (2) & \text { if } t \text { is odd, } \\
\hat{h}([-4,2 t]) \geq \frac{187}{384} \log \left(16+t^{2}\right)-\frac{41}{16} \log (2) & \text { in any case, } \\
\hat{h}([-4,2 t]) \leq \frac{193}{384} \log \left(16+t^{2}\right)+\frac{137}{768} \log (2) & \text { in any case. }
\end{array}
$$

We now have sufficiently good estimates to prove some of our observations when the rank is 1 .

\section{Solving Diophantine problems in rank 1}

In this section, we will prove most of our observations concerning the structure of $C_{t}(\mathbb{Q})$ and the integral points both on $C_{t}$ and $Q_{t}$.

Theorem 10.1. Let $t$ be an integer defining a simplest quartic field, and $C_{t}$ be the associated elliptic curve. Then the point $[-4,2 t]$ can always be in a system of generators. In particular, if the rank of $C_{t}$ is one,

$$
C_{t}(\mathbb{Q})=\langle[0,0],[-4,2 t]\rangle \text {. }
$$

Proof. Assume that $G_{1}=[-4,2 t]$ cannot be in a system of generators. This means that there exist $P \in C_{t}(\mathbb{Q}), \varepsilon \in\{0,1\}$ and $n \in \mathbb{Z}$ such that

$$
G_{1}=n P+\varepsilon[0,0] \text {. }
$$

So the canonical height of $G_{1}$ equals the canonical height of $n P$ and

$$
n^{2}=\frac{\hat{h}\left(G_{1}\right)}{\hat{h}(P)} .
$$

The estimates obtained above can now be used to bound $n^{2}$. If $t \geq 257$

$$
n^{2} \leq \frac{\frac{193}{384} \log \left(16+t^{2}\right)+\frac{137}{768} \log (2)}{\frac{1}{8} \log \left(16+t^{2}\right)+0.38-\log (2)}
$$


Since this function decreases with $t$, it is easy to prove that

$$
n^{2} \leq 5.31 \text { if } t \geq 257 \text {. }
$$

The remaining cases, namely $n=2$ or $t \leq 256$, can be computed by hand.

Let us now concentrate on integral points.

When the rank is one, the structure of the Mordell-Weil group is known, so we are using it to find integral points on $C_{t}$. If $P$ is an integral point then there exist $\varepsilon \in\{0,1\}$ and $n \in \mathbb{Z}$ such that

$$
P=n G_{1}+\varepsilon[0,0] \text {. }
$$

The strategy is the same as above, namely we are using the bounds on canonical heights to deduce an upper bound on $n$. But, in this case, we need an upper bound for the canonical height of any integral point. Using Silverman's bounds, this means that we need an upper bound for the naïve height of any integral point. This is of course not possible unless we have an explicit version of Siegel's theorem. In the case of simplest cubic fields, we proved by an other means that there are no integral points in the connected component of the point at inifinity of the curve. This cannot be done for simplest quartic fields for any $t$. However, it can be done if $t$ is odd. For this, we will use the following lemma.

Lemma 10.2. Let $E$ be an elliptic curve defined over $\mathbb{Q}$ and $P$ be a point on $E(\mathbb{Q})$ which is not integral. Then none of the multiples of $P$ are integral.

Proof. We just give the idea of the proof. Let $p$ be a prime number dividing the denominator of the coordinates of $P$. The reduction of $P$ modulo $p$ is the point at infinity on the reduced curve. So all multiples of $P$ are also the point at infinity on the reduced curve. Thus their denominators are also divisible by $p$.

Theorem 10.3. Let $t$ be an odd number defining a simplest quartic field. Assume that the elliptic curve $C_{t}$ has rank 1, then the only integral points on $C_{t}$ are $[0,0]$ and $[-4, \pm 2 t]$.

Proof. Let $P$ be an integral point on $C_{t}$. Then, there exist $\varepsilon \in\{0,1\}$ and $n \in \mathbb{Z}$ such that

$$
P=n G_{1}+\varepsilon[0,0] .
$$

Three cases can occur

- $n$ is even and $\varepsilon=0$. In this case, $P$ is a multiple of $2[-4,2 t]$ which is never an integral point if $t \neq 4,8$. Lemma 10.2 then ensures that $P$ is not an integral point.

- $n$ is odd and $\varepsilon=1$. Again $P$ is a multiple of $[-4,2 t]+[0,0]$ which is not an integral point and we use Lemma 10.2. 
- $n$ is odd and $\varepsilon=0$ or $n$ is even and $\varepsilon=1$. In this case, $P$ is not in the connected component of the point at infinity of $C_{t}(\mathbb{R})$ so its $x$-coordinate is bounded

$$
-\sqrt{16+t^{2}} \leq x(P) \leq 0 .
$$

The method using canonical heights can then be applied. Thanks to Proposition 8.1, the canonical height of such a point is bounded as follows

$$
\hat{h}(P) \leq h(P)+\frac{1}{2} \log \left(16+t^{2}\right)+4.08 \leq \log \left(16+t^{2}\right)+4.08 .
$$

Using the lower bound for the canonical height of $[-4,2 t]$ obtained in section 9 , we deduce that

$$
n^{2} \leq \frac{\log \left(16+t^{2}\right)+4.08}{\frac{187}{384} \log \left(16+t^{2}\right)-\frac{1}{16} \log (2)} .
$$

Again, the function is decreasing and

$$
n^{2} \leq 3.9 \text { if } t \geq 10 \text {. }
$$

So only $n=0,1$ or -1 can provide integral points.

Remark. The second case cannot be treated if $t$ is even because $[-4,2 t]+$ $[0,0]$ is an integral point.

We deduce the following corollary from this theorem and Proposition 3.3

Corollary 10.4. Let $t$ be an odd number defining a simplest quartic field such that $Q_{t}$ has rank 1, then the only integral points on $Q_{t}$ are $[0, \pm 1]$.

In fact, we can prove this also when $t$ is even thanks to the following lemma.

Lemma 10.5. Let $t$ be an odd number defining a simplest quartic field. Let $P=[X, Y]$ be an integral point on $Q_{t}$ such that $Y \leq 0$. Then $\varphi(P)+[0,0]$ is an integral point on $C_{t}$ whose $x$-coordinate is bounded by $t^{2}$.

Proof. The $x$-coordinate of $\varphi(P)+[0,0]$ equals $2 Y+2 X^{2}-t X-2$ and $Y=-\sqrt{X^{4}-t X^{3}-6 X^{2}+t X+1}$. Thus, it is sufficient to prove that

$$
\left(2 X^{2}-t X-2-t^{2}\right)^{2}-4\left(X^{4}-t X^{3}-6 X^{2}+t X+1\right) \leq 0 .
$$

This polynomial is a degree 2 polynomial and it is easy to prove that it is negative outside of ] $-\frac{n}{3}-1, n+1\left[\right.$. Within this range, $2 X^{2}-t X-2-t^{2}$ is always negative which achieves the proof.

We can now prove the following theorem

Theorem 10.6. Let $t$ be an integer defining a simplest quartic field such that $Q_{t}$ has rank 1, then the only integral points on $Q_{t}$ are $[0, \pm 1]$. 
Proof. Thanks to Lemma 10.5, it is sufficient to find all integral points on $C_{t}$ whose naïve height is less than or equal to $t^{2}$. Let $P$ be such an integral point. Proposition 8.1 provides an upper bound for its canonical height.

$$
\hat{h}(P) \leq h(P)+\frac{1}{2} \log \left(16+t^{2}\right)+4.08 \leq \frac{3}{2} \log \left(16+t^{2}\right)+4.08 .
$$

If $P=n[-4,2 t]+\varepsilon[0,0]$, then

$$
n^{2} \leq \frac{\frac{3}{2} \log \left(16+t^{2}\right)+4.08}{\frac{187}{384} \log \left(16+t^{2}\right)-\frac{41}{16} \log (2)} .
$$

As in the previous cases, the function is decreasing and we deduce that

$$
n^{2} \leq 8.92 \text { if } t \geq 33 \text {. }
$$

The remaining cases, namely $n=2$ or $t \leq 32$, can easily be done by hand.

We are now interested in the last observation in section 4 which will provide a subfamily with a rank of at least 2 .

\section{A subfamily with a rank of at least 2}

During our numerical experiments, we noticed that -3 is sometimes the $x$-coordinate of an integral point on $Q_{t}$. It is in fact not difficult to prove that

$$
[-3, \ldots] \in Q_{t}(\mathbb{Z}) \Longleftrightarrow t=6 k^{2}+2 k-1 \text { with } k \in \mathbb{Z} .
$$

In this case, there are new integral points on $C_{t}(\mathbb{Q})$. One of them is of course given by $\varphi([-3,2+12 k])+[0,0]$. These new points are the following and their opposites.

$$
\begin{aligned}
G_{2} & =\left[-2 k^{2}+2 k-1,4(k+1)\left(2 k^{2}-2 k+1\right)\right], \\
G_{2}+[0,0] & =\left[18 k^{2}+30 k+17,4(k+1)\left(18 k^{2}+30 k+17\right)\right], \\
G_{1}+G_{2} & =\left[9\left(2 k^{2}-2 k+1\right), 12(3 k-2)\left(2 k^{2}-2 k+1\right)\right] .
\end{aligned}
$$

Since $t$ is odd and $G_{2}$ is an integral point, Theorem 10.3 ensures that the rank is at least 2. The aim of the rest of this paper is to generalize the results obtained in rank 1 to the case of rank 2 using this subfamily. Let us first consider the structure of the Mordell-Weil group.

\section{Case of rank 2: generators}

The infinite descent generalizes to higher ranks the method we used to prove that $G_{1}$ can always be in a system of generators. Let us first recall the principle of this method.

Suppose that $P_{1} \ldots P_{r}$ generate a subgroup of the free part of the MordellWeil group of full rank and denote by $n$ the index of this subgroup. If $n=1$, this provides a basis. Let $R$ be the regulator of the curve (i. e. the 
elliptic regulator of a basis $B$ of the free part of the Mordell-Weil group), then we have

$$
n^{2} R=R\left(P_{1} \ldots P_{r}\right) .
$$

Since the regulator is roughly of the same order of magnitude as the product of the canonical heights of the basis $B$, it can be bounded using Proposition 8.3. So that $n$ can be bounded. In [13], Siksek specifies this idea by the following theorem (written here only in the case of rank 2 and base field Q).

Theorem 12.1 (Siksek). Let $E$ be an elliptic curve defined over $\mathbb{Q}$ of rank 2. Suppose that $E(\mathbb{Q})$ contains no point of infinite order with a canonical height less than some positive real number $\lambda$. Suppose that $P_{1}$ and $P_{2}$ generate a subgroup of the free part of the Mordell-Weil group of full rank and denote by $n$ the index of this subgroup. Then we have

$$
n \leq \frac{2}{\sqrt{3}} \frac{R\left(G_{1}, G_{2}\right)^{\frac{1}{2}}}{\lambda} .
$$

As explained above, the infinite descent is based on canonical heights. Thus, we need to approximate the canonical heights of the points involved in our problem.

Proposition 12.2. Let $k$ be an integer such that $t=6 k^{2}+2 k-1$ defines a simplest quartic field and such that $|k| \geq 27$, then we have

$$
\begin{aligned}
& 0.96 \log (t) \leq \hat{h}\left(G_{1}\right) \leq 1.02 \log (t) \\
& 0.47 \log (t) \leq \hat{h}\left(G_{2}\right) \leq 0.56 \log (t) \\
& 0.47 \log (t) \leq \hat{h}\left(G_{1}+G_{2}\right) \leq 0.54 \log (t)
\end{aligned}
$$

Proof. The first estimate is a direct consequence of the estimates given in section 9. Estimates for the canonical height of $G_{2}$ and $G_{1}+G_{2}$ are obtained in the same way, namely using the first four terms of the Tate series for the Archimedean contribution. Non-Archimedean contributions are given by formula (2), knowing that $t$ is odd and that the gcd of $a, b$ and $16+t^{2}$ is exactly $2 k^{2}-2 k+1$ both for $G_{2}$ and $G_{1}+G_{2}$.

We can now prove the following theorem

Theorem 12.3. Let $k$ be an integer such that $t=6 k^{2}+2 k-1$ defines a simplest quartic field. Then the points $G_{1}=[-4,2 t]$ and $G_{2}=$ $\left[-2 k^{2}+2 k-1,4(k+1)\left(2 k^{2}-2 k+1\right)\right]$ can always be in a system of generators. In particular, if the rank of $C_{t}$ is exactly 2 , we have

$$
C_{t}(\mathbb{Q})=\left\langle G_{1}, G_{2},[0,0]\right\rangle .
$$

Proof. In order to apply Siksek's theorem, we need an estimate of

$$
R\left(G_{1}, G_{2}\right)=\hat{h}\left(G_{1}\right) \hat{h}\left(G_{2}\right)-\left\langle G_{1}, G_{2}\right\rangle^{2}
$$


with $\left\langle G_{1}, G_{2}\right\rangle=\frac{1}{2}\left(\hat{h}\left(G_{1}+G_{2}\right)-\hat{h}\left(G_{1}\right)-h\left(G_{2}\right)\right)$. Proposition 12.2 provides these estimates

$$
\begin{aligned}
-0.56 \log (t) \leq\left\langle G_{1}, G_{2}\right\rangle & \leq-0.44 \log (t) \\
R\left(G_{1}, G_{2}\right) & \leq 0.39(\log (t))^{2}
\end{aligned}
$$

Siksek's theorem then ensures that if $G_{1}$ and $G_{2}$ generate a subgroup of index $n$ of the free part of the Mordell-Weil group, then

$$
n \leq \frac{2}{\sqrt{3}} \frac{R\left(G_{1}, G_{2}\right)^{\frac{1}{2}}}{\lambda},
$$

with $\hat{h}(P) \geq \lambda$ for any point $P$ in the free part of the Mordell-Weil group. The estimates obtained in Propositions 12.2 and 8.3 imply that, for any $k$ such that $|k| \geq 27$,

$$
n \leq \frac{2}{\sqrt{3}} \frac{\sqrt{0.39} \log (t)}{0.38+\frac{1}{8} \log \left(16+t^{2}\right)} \leq \frac{2}{\sqrt{3}} \frac{\sqrt{0.39} \log (t)}{\frac{1}{4} \log (t)} \leq 2.9 .
$$

The case $n=2$ must be treated by hand. For this, it is sufficient to prove that there are no point $Q \in C_{t}(\mathbb{Q})$ and integers $\varepsilon_{1}$ and $\varepsilon_{2}$ in $\{0,1\}$ such that

$$
\varepsilon_{1} G_{1}+\varepsilon_{2} G_{2}=2 Q .
$$

This is not difficult because $G_{1}, G_{2}$ and $G_{1}+G_{2}$ are integral points, so $Q$ must be an integral point because of Lemma 10.2. Looking at the numerator and denominator of the double of any integral point modulo 8 shows that such a double is not an integral point.

Finally, the cases with $k \leq 26$ can be treated by hand (i. e. using magma).

The structure of the Mordell-Weil rank is now completely determined and can be used to find integral points.

\section{Case of rank 2: integral points}

The situation is the same as in rank one, namely we do not have any bound for the naïve height for integral points on $C_{t}(\mathbb{Q})$, so it is not possible, with our method, to determine all integral points on $C_{t}$. However, we can use the same trick to determine all integral points on $Q_{t}$.

Theorem 13.1. Let $k$ be an integer such that $t=6 k^{2}+2 k-1$ defines a simplest quartic field. Suppose that $Q_{t}$ has rank 2, then the only integral points on $Q_{t}$ are $[0, \pm 1]$ and $[-3, \pm(2+12 k)]$.

Proof. Thanks to Lemma 10.5, it is sufficient to find all integral points on $C_{t}$ whose naïve height is less than or equal to $t^{2}$. Let $P$ be such an 
integral point. We have an upper bound for its canonical height provided by Proposition 8.1 .

$$
\hat{h}(P) \leq \frac{3}{2} \log \left(16+t^{2}\right)+4.08 .
$$

Theorem 12.3 implies that there are integers $n_{1}$ and $n_{2}$ and $\varepsilon \in\{0,1\}$ such that

$$
P=n_{1} G_{1}+n_{2} G_{2}+\varepsilon[0,0] .
$$

using the properties of the canonical height, we deduce that

$$
\hat{h}(P)=n_{1}^{2} \hat{h}\left(G_{1}\right)+n_{2}^{2} \hat{h}\left(G_{2}\right)+2 n_{1} n_{2}\left\langle G_{1}, G_{2}\right\rangle .
$$

We know, thanks to Proposition 12.2, that $\left\langle G_{1}, G_{2}\right\rangle$ is negative and that $\hat{h}\left(G_{1}\right) \geq \hat{h}\left(G_{2}\right)$. Hence it is easy to conclude if $n_{1} n_{2}$ is non-positive. Indeed, we have

$$
\hat{h}(P) \geq\left(n_{1}^{2}+n_{2}^{2}\right) \hat{h}\left(G_{2}\right) .
$$

So, if $|k| \geq 27$, we have

$$
\begin{aligned}
n_{1}^{2}+n_{2}^{2} & \leq \frac{\frac{3}{2} \log \left(16+t^{2}\right)+4.08}{0.47 \log (t)} \\
& \leq 7.5
\end{aligned}
$$

This proves that both $\left|n_{1}\right|$ and $\left|n_{2}\right|$ are less than or equal to 2 , but not at the same time.

If $n_{1} n_{2}$ is positive, it is more subtle. In this case we especially need precise approximations of Proposition 12.2. If $|k| \geq 27$, we have

$$
\begin{aligned}
\hat{h}(P) & \geq 0.96 \log (t) n_{1}^{2}+0.47 \log (t) n_{2}^{2}-1.11 \log (t) n_{1} n_{2} \\
& \geq 0.47\left(2.04 n_{1}^{2}+n_{2}^{2}+2.38 n_{1} n_{2}\right) \log (t) \\
& \geq 0.47\left(0.62 n_{1}^{2}+\left(1.19 n_{1}-n_{2}\right)^{2}\right) \log (t)
\end{aligned}
$$

Using the upper bound on $\hat{h}(P)$ given by Silverman, we deduce

$$
\begin{aligned}
\left(0.62 n_{1}^{2}+\left(1.19 n_{1}^{2}-n_{2}\right)^{2}\right) & \leq \frac{\frac{3}{2} \log \left(16+t^{2}\right)+4.08}{0.47 \log (t)} \\
& \leq 7.5
\end{aligned}
$$

We assume, without loss of generality, that $n_{1}$ and $n_{2}$ are both positive. It is easy to deduce that $n_{1}$ must be less than or equal to 3 and that

$$
\begin{aligned}
& n_{1}=1 \quad \Longrightarrow \quad n_{2} \leq 3 \\
& n_{1}=2 \quad \Longrightarrow \quad n_{2} \leq 4 \\
& n_{1}=3 \quad \Longrightarrow \quad n_{2}=3 \text { or } 4
\end{aligned}
$$

The remaining cases must be done by hand; for $|k|<27$ we used magma. For small values of $n_{1}$ and $n_{2}$, we are again using canonical heights. Let 
us treat, for instance, the case $n_{1}=2$ and $n_{2}=-1$. The bounds given in Proposition 12.2 ensure that

$$
\hat{h}\left(G_{1}-2 G_{2}\right) \geq 4.34 \log (t) .
$$

If $G_{1}-2 G_{2}$ is an integral point, its naïve height equals the logarithm of its $x$-coordinate, so, using Proposition 8.1, we have

$$
\hat{h}\left(G_{1}-2 G_{2}\right) \leq \log (x(P))+\frac{1}{2} \log \left(16+t^{2}\right)+4.08
$$

with

$$
x(P)=-4\left(\frac{24 k^{5}+60 k^{4}+24 k^{3}-48 k^{2}-54 k-13}{20 k^{4}+56 k^{3}+88 k^{2}+76 k+29}\right)^{2} .
$$

These two bounds are incompatible so $G_{1}-2 G_{2}$ is never an integral point. In some cases, Silverman's bounds are not precise enough and thus we used bounds obtained by Tate's series.

Finally, this proves that the only integral points having their $x$-coordinate less than or equal to $t^{2}$ on $C_{t}$ are $[0,0], G_{1}, G_{2}, G_{1}+G_{2}, G_{2}+[0,0]$ and $G_{1}+2 G_{2}$ if $k \equiv-1 \bmod 5$ and their opposites. Using the reciprocal map of $\varphi$, it is easy to find all integral points on $Q_{t}$.

\section{Conclusion}

As in the case of simplest cubic fields, we succeeded in proving that the point $[-4,2 t]$ can always be in a system of generators of $C_{t}(\mathbb{Q})$. We also succeeded in generalizing this to the rank 2 case. This is not surprising since it is based on the infinite descent method . Moreover, it is almost sure that it will also work with other families or with higher ranks assuming, of course, that explicit generators exist and are known.

On the contrary, we encountered difficulties in solving the problem of integral points on $C_{t}$, even in rank 1. This is due to the fact that we do not know any bound on the naïve height of integral points. This difficulty can be overcome in some specific situations, as in the case of simplest cubic fields or of simplest quartic fields when the parameter is odd. In fact, we noticed that the method used for simplest cubic fields in rank 1 will be successful for any family of torsion-free curves of rank 1 .

However, we were able to give exactly all integral points on the original model of the curve both in the case of rank 1 and in the case of a subfamily of curves of rank 2 . 


\section{References}

[1] H. Cohen, A Course in Computational Algebraic Number Theory. Graduate Texts in Math. 138, Springer-Verlag (1993).

[2] J. Cremona, M. Prickett, S. Siksek, Height difference bounds for elliptic curves over number fields. Journal of Number Theory 116 (2006), 42-68.

[3] J. Cremona, S. Siksek, Computing a Lower Bound for the Canonical Height on Elliptic Curves over $\mathbb{Q}$. Algorithmic Number Theory, 7th International Symposium, ANTS-VII, LNCS 4076 (2006) 275-286.

[4] S. Duquesne, Integral points on elliptic curves defined by simplest cubic fields. Exp. Math. 10:1 (2001), 91-102.

[5] M. N. Gras, Table numérique du nombre de classes et des unités des extensions cycliques réelles de degré 4 de $\mathbb{Q}$. Publ. Math. Fac. Sci. Besancon, fasc 2, (1977/1978).

[6] E. Halberstadt, Signes locaux des courbes elliptiques en 2 et 3. C. R. Acad. Sci. Paris Sér. I Math. 326:9 (1998), 1047-1052.

[7] H. K. KIM, Evaluation of zeta functions at $s=-1$ of the simplest quartic fields. Proceedings of the 2003 Nagoya Conference "Yokoi-Chowla Conjecture and Related Problems", Saga Univ., Saga (2004), 63-73.

[8] A. J. LazARus, Class numbers of simplest quartic fields. Number theory (Banff, AB, 1988), de Gruyter, Berlin (1990), 313-323.

[9] A. J. Lazarus, On the class number and unit index of simplest quartic fields. Nagoya Math. J. 121 (1991), 1-13.

[10] S. Louboutin, The simplest quartic fields with ideal class groups of exponents less than or equal to 2. J. Math. Soc. Japan 56:3 (2004), 717-727.

[11] P. Olajos, Power integral bases in the family of simplest quartic fields. Experiment. Math. 14:2 (2005), 129-132.

[12] O. Rizzo, Average root numbers for a nonconstant family of elliptic curves. Compositio Math. 136:1 (2003), 1-23.

[13] S. SiKSEK, Infinite descent on elliptic curves. Rocky Mountain J. Math. 25:4 (1995), 15011538.

[14] J. H. Silverman, The arithmetic of elliptic curves. Graduate Texts in Mathematics 106, Springer-Verlag (1986).quand tout sera fini

[15] J. H. Silverman, Computing heights on elliptic curves. Math. Comp. 51 (1988), 339-358.

[16] J. H. Silverman, The difference between the Weil height and the canonical height on elliptic curves. Math. Comp. 55 (1990), 723-743.

Sylvain Duquesne

Université Montpellier II

Laboratoire I3M (UMR 5149) et LIRMM (UMR 5506)

CC 051, Place Eugène Bataillon

34005 Montpellier Cedex, France

E-mail : duquesne@math.univ-montp2.fr 